\title{
Introducción de verbos \\ inacusativos en la clase de español \\ como lengua extranjera ${ }^{1}$
}

\section{(Introduction of Unaccusative Verbs in the Spanish as a Foreign Language Classroom)}

\section{Ming Yang ${ }^{2}$}

Universidad de Estudios Internacionales de Shanghái (SISU), China

\section{Resumen}

La transitividad verbal supone para los estudiantes chinos una gran dificultad durante su aprendizaje de Español como Lengua Extranjera (E/ LE). Les resulta confuso el uso de los verbos de comportamiento inestable. En la tradición gramatical, se distinguen verbos transitivos, intransitivos e impersonales. La Hipótesis Inacusativa (Perlmutter, 1978) divide los verbos intransitivos en inergativos e inacusativos. En este artículo se propone que el orden del sujeto y predicado, y el uso del participio pasivo o la voz media en español, se entienden con más facilidad con la introducción del concepto de verbos inacusativos, analizados desde la interfaz léxicosintaxis. Ofrece una nueva perspectiva para la enseñanza de E/LE accesible para los estudiantes chinos.

\begin{abstract}
Verbal transitivity always supposes a great difficulty for Chinese students of Spanish as foreign language (SFL). Verbs with unstable valency behavior cause confusion. According to traditional grammar, there are transitive, intransitive, or impersonal verbs. The Unaccusative Hypothesis (Perlmutter,
\end{abstract}

1 Recibido: 22 de julio de 2018; aceptado: 11 de febrero de 2019.

2 Departamento de Español de la Facultad de Estudios Europeos y Latinoamericanos. Correo electrónico: yangming @ shisu.edu.cn. El autor agradece a los evaluadores por las sugerencias y correcciones planteadas. Solo al autor se atribuyen los errores que estas páginas puedan contener.

Letras 65 (2019), ISSN 1409-424X; EISSN 2215-4094

Doi: http://dx.doi.org/10.15359/rl.1-65.5

www.revistas.una.ac.cr/index.php/letras 
1971) classifies intransitive verbs as unaccusative or unergative. In this article we propose that word order of the subject and predicate, and the absolute passive participle structure and middle voice, in Spanish can be understood more easily with the introduction of the concept of unaccusative verbs analyzed with the lexical-syntactic interface, thus offering a new perspective for the teaching of SFL to Chinese students.

Palabras clave: estructura argumental, interfaz léxico-sintaxis, verbos inacusativos, alternancias verbales

Keywords: argument structure, lexicon-syntax interface, unaccusative verbs, verb alternations

\section{Introducción: la estructura argumental}

En numerosas lenguas naturales, como en el caso de las más habladas en el mundo - el chino mandarín, el español y el inglés - la estructura básica y más frecuente de un enunciado es la de sujetopredicado. Véanse unos ejemplos con el verbo open (abrir) ${ }^{3}$ y sus equivalentes en español y en chino:

1) Inglés: The door opened.

Español: La puerta se abrió.

Chino: 门开了。

men-kai-le

puerta-abierto-le (partícula aspectual)

2) Inglés: John opened the door

Español: Juan abrió la puerta

Chino: 约翰开了门。

Yuehan-kai-le-men

Juan-abrir-le-puerta

3 Charles J. Fillmore, «The Case for Case», Universals in Linguistic Theory (London: Holt, Rinehart and Winston, 1968) 1-25. 
3) Inglés: the wind opened the door

Español: el viento abrió

Chino: 风吹开了门。

Feng-chui-kai-le-men

viento-soplar-abierto-le-puerta

4) Inglés: John opened the door with a chisel

Español: Juan abrió la puerta con un cincel

Chino: 约翰用丵子打开了门。

Yuehan-yong-zaozi-dakai-le-men

Juan-usar(con)-cincel-abrir-le-puerta

Encontramos un problema típico de las alternancias de la estructura argumental. Con tales ejemplos se nota una relación sintáctica sujeto-predicado desde un punto de vista de la gramática tradicional. Se observa también que en 2) 3) y 4) el verbo open lleva the door como objeto y que el verbo es capaz de pasar su acción al objeto. Estos ejemplos ilustran la generalización de que, por un lado, en oraciones declarativas con sujetos y objetos nominales en el orden dominante (en la mayoría de las lenguas) el sujeto precede al objeto; por el otro, que el sujeto gramatical del verbo puede ser indistintamente agente en 2) y 4), tema como en 1), o fuerza en 3). Es evidente que las relaciones sintácticas no son identificables con las nociones de sujeto y predicado. De ello Fillmore infiere la existencia de diversos tiposde frase o sintagma nominal -casos-introducidos categorialmente en relación con cada verbo. Como es sabido, el predicado denota una acción, un estado o un proceso. En los cuatro ejemplos hay complementos semánticamente requeridos por el predicado, denominados argumentos o actantes (Real Academia Española, Nueva gramática de la lengua española (Manual), en adelante llamada el Manual: 15). Pero también se puede observar, comparando 2) y 4), que hay complementos, denominados adjuntos no seleccionados por el predicado, como with a chisel en 4), que expresa un instrumento. Desde el punto 
de vista semántico, la oración se divide en predicado y argumentos. Estos son los participantes en la acción, estado o proceso que denota el predicado. El conjunto de argumentos que el propio predicado ocupa para complementar su significado se llama estructura argumental (EA). La introducción del concepto de la EA conlleva identificar el verbo como el núcleo de la oración y establecer un haz de relaciones semánticas con los sintagmas nominales (SN) que lo acompañan.

Con la teoría de argumentos, clasificamos los predicados por su valencia. El término valencia, prestado de la química, indica el número que expresa la capacidad de combinación de un elemento químico con otros para formar un compuesto; en lingüística, se refiere al número de argumentos que exigen los predicados. Los predicados avalentes no toman ningún argumento; los monovalentes requieren un único argumento; los bivalentes exigen dos; y, por último, los trivalentes toman tres. Vamos a ver los ejemplos del Manual ${ }^{4}$.

5) llover, nevar, amanecer $\varphi$

6) El guarda duerme.

7) $\quad$ Ana decidió no acudir a la boda.

8) El señor ministro entregó los premios ayer a los galardonados en una brillante ceremonia.

Se subrayan los argumentos en 5), 6), 7) y 8). Según la estructura temática (theta grid), introducida en las entradas léxicas por Chomsky ${ }^{5}$ siguiendo las ideas de Stowell ${ }^{6}$, podemos analizar los argumentos de $5), 6), 7)$ y 8) de la siguiente manera:

$4 \quad$ Nueva gramática de la lengua española, Manual (Madrid: Espasa, 2010), 17.

5 Noam Chomsky, Lectures on Government and Binding (Dordrecht: Foris, 1981).

6 Tim Stowell, Origins of Phrase Structure (Cambridge, MA: MIT Press, 1981). 
9) amanecer: $\langle\varphi\rangle$

10) dormir: <experimentante>

11) decidir: <agente, tema>

12) entregar: <agente, tema, destinatario>

A continuación citamos ejemplos de la Gramática descriptiva de la lengua española ${ }^{7}$ y los datos que aparecen en Perspectivas de sintaxis formal':

13) a. *Guillermo hace.

b. *Pablo puso en el corral.

c. *Pablo puso los pollos.

d. *Pablo puso.

14) a. Juan llenó el depósito (de agua).

b. Chomsky habló (de lingüística).

Los estudiantes chinos de español pueden notar la agramaticalidad de 13a) 13b) y 13d), y con base en los conocimientos adquiridos de la gramática tradicional, fácilmente entienden que los enunciados mencionados son agramaticales debido a la ausencia delobjeto directo de un verbo transitivo como poner o hacer. Pero pocos estudiantes entenderán la agramaticalidad de 13c), ya que en este caso el verbo transitivo tiene complemento directo. Lo mismo sucede en el aprendizaje de verbos como llenar y hablar. Podemos decir que el conjunto de argumentos de un predicado supone la definición lógica y conceptual del verbo. Así, las acciones de los verbos poner, llenar y hablar no se

7 Ignacio Bosque y Violeta Demonte, Gramática descriptiva de la lengua española (Madrid: Espasa, 1999).

8 Ángel J. Gallego, Perspectivas de sintaxis formal (Madrid: Ediciones Akal, 2015). 
pueden concebir sin la participación de los sintagmas preposicionales en el corral, de agua, y de lingüística. Esta conciencia que tiene un nativo acerca del comportamiento sintáctico del predicado constituye una parte importante de conocimiento durante el aprendizaje de E/LE.

\section{La interfaz léxico-sintaxis: la relación entre significado y estructura}

Según la gramática generativa, el lenguaje es una capacidad biológica del ser humano, exclusiva de él y diferente de sus demás capacidades, con muchos elementos comunes a todas las lenguas. Uno de los supuestos por los que más se caracteriza la corriente generativista es el carácter innato de la facultad lingüística. Como el mismo Chomsky (1995) reitera en AMinimalist Programfor Linguistic Theory [Programa minimalista para la teoría lingüística]:

Language and its use have been studied from varied points of view. One approach, assumed here, takes language to be part of the natural world. The human brain provides an array of capacities that enter into the use and understanding of language (the language faculty); $[\ldots]^{9}$

Escandell (2011) menciona en Invitación a la lingüística tres etapas históricas del generativismo chomskyano ${ }^{10}$.

- La Teoría estándar, comienza con Estructuras Sintácticas (1957).

- $\quad$ La Teoría de principios y parámetros (P\&P), también conocida como Government and Binding (rección y ligamiento) comienza en 1981 con Lectures on GB.

- El más actual, el Programa minimalista, afina la Teoría de P\&P.

9 Noam Chomsky, The Minimalist Program (Cambridge, MA: MIT, 1995) 167.

10 M. V. Escandell Vidal, Invitación a la lingüística (Madrid: Editorial Universitaria Ramón Areces, 2011) 285 . 
El modelo de Principios y Parámetros considera la gramática como «un conjunto restringido de principios generales, comunes a todas las lenguas, que lleva asociado un conjunto también restringido de parámetros responsables de la variación entre las lenguas ${ }^{11}{ }$.

Con la teoría de P \& P, la interfaz léxico-sintaxis recibe cada día mayor atención y muchas investigaciones se realizan en torno a la relación entre la representación léxica de los predicados y su realización sintáctica. Como Levin opina en la introducción de su investigación English Verb Classes and Alternations, «el comportamiento de un verbo, sobre todo con respecto a la expresión e interpretación de sus argumentos, depende en gran medida de su significado; por consiguiente, se aprovecha el comportamiento verbal para comprobar su vinculación con los aspectos pertinentes del significado verbal» ${ }^{12}$.

Según la teoría generativista, la relación entre una estructura superficial (E-S) y una estructura profunda (E-P), en que esta corresponde a la representación más básica de la oración en la que aparecen sus elementos significativos, mientras en aquella se encuentra el enunciado tal como ha sido emitido. La oración nace en la E-P y experimenta una serie de restricciones hasta llegar a la E-S, nivel en el cual el enunciado obtiene su forma fonética $(\mathrm{FF})$ y su forma lógica (FL). Para Bloomfield ${ }^{13}$, el léxico es un apéndice de la gramática, una lista de las irregularidades básicas, para construir unidades gramaticales más grandes en el marco de la sintaxis (sistema computacional). El léxico y el sistema computacional constituyen nuestro sistema lingüístico. La FF y la FL llegan a los dos sistemas externos, que son el articulatorio-perceptivo (A-P) y el conceptual-intencional (C-I), correspondientemente. En el modelo de Principios y parámetros, el Principio de proyección de Chomsky ${ }^{14}$ garantiza la compatibilidad entre la información léxica y la representación sintáctica del significado.

11 Escandell Vidal, 289.

12 Beth Levin, English Verb Classes and Alternations: A Preliminary Investigation (Cambridge, MA: University of Chicago Press, 1993).

13 L. Bloomfield, Language (Cambridge, MA: University of Chicago Press, 1933).

14 Chomsky (1981). 


\section{La hipótesis de la inacusatividad: verbos inacusativos}

Según el principio de proyección, la E-P constituye una representación sintáctica de las propiedades argumentales de los verbos. Fillmore ${ }^{15}$ propone tres formas proposicionales, dejando de lado la complejidad de la construcción pasiva y simplificando los tipos de argumentos hasta que solo queden el agente y el objeto:
(a) $\mathrm{V}+\mathrm{A}$
(b) $\mathrm{V}+\mathrm{O}+\mathrm{A}$
(c) $\mathrm{V}+\mathrm{O}$

Estos tres prototipos corresponden a oraciones intransitivas con sujetos agentivos, oraciones transitivas con sujetos agentivos y oraciones intransitivas con sujetos no agentivos. Comparando los tipos (a) y (c), Fillmore toma en cuenta las distintas características semánticas y sintácticas de esta clase verbal heterogénea, los verbos intransitivos. La distinción entre dos clases de verbos intransitivos se debe a Perlmutter ${ }^{16}$ con la hipótesis de la inacusatividad, que divide la clase de verbos intransitivos entre inergativos e inacusativos, según si su único argumento es agente o no. La hipótesis se formuló en el contexto de la gramática relacional y fue adoptada por Burzio ${ }^{17}$ en Italian Syntax dentro del marco de la Teoría de rección y ligamiento. Para Levin ${ }^{18}$, es una hipótesis sintáctica que parte de la dicotomía entre verbos inergativos e inacusativos asociados con diferentes configuraciones sintácticas profundas.

15 Charles J. Fillmore, «The Case for Case», Universals in Linguistic Theory (Londres: Holt, Rinehart and Winston, 1968).

16 David M. Perlmutter, «Impersonal Passives and the Unaccusative Hypothesis», Annual Meeting of the Berkeley Linguistics Society (UC Berkeley, 1978).

17 L. Burzio, Italian Syntax. A Government-binding Approach (Dordrecht, Reidel: 1981).

18 Beth Levin y Malka Rappaport Hovav, Unaccusativity: At the Syntax-lexical Semantics Interface (MA: MIT, 1995). 
Burzio demuestra que hay una diferencia en el comportamiento de los verbos intransitivos italianos arrivare y telefonare, que muestran los siguientes ejemplos tomados de la Gramática descriptiva ${ }^{19}$ :

15) a. Arriveranno molti esperti.

b. Ne arriveranno molti.

16) a. Telefoneranno molti esperti.

b. *Ne telefoneranno molti.

Como se observaen 15) y 16), los verbos como arrivare permiten construcciones con un sujeto posverbal cuantificado pronominalizado por el clítico $n e$; eso no es posible con verbos como telefonare. La diferencia entre estos dos tipos de verbos italianos se ve reforzada por otro hecho morfosintáctico: su compatibilidad, o no con essere como verbo auxiliar perfectivo.

17) Sonno/*Hanno arrivati molti esperti.

18) Hanno/*Sonno telefonato molti esperti.

Estos dos hechos son pruebas prototípicas para la inacusatividad en la lengua italiana. En español, estos dos fenómenos lingüísticos no existen, aunque sí estuvo presente en él hasta el siglo xvi la distinción entre ser y haber como auxiliares perfectivos ${ }^{20}$. Debido a la ausencia del clítico partitivo, y la presencia de un solo auxiliar (haber) en los tiempos compuestos, las evidencias de la inacusatividad en español no son tan rotundos como en italiano.

En cuanto al chino, la inacusatividad fue tema de investigaciones en la década de 1980 en el círculo lingüístico occidental ${ }^{21}$. El

19 Bosque y Demonte.

20 Bosque y Demonte.

21 Andrè Martinet, Syntaxe Générale (Madrid: Editorial Gredos, 1987). 
chino mandarín muestra perfectamente el caso de una construcción inacusativa (ergativa), como se observa en los siguientes ejemplos:

19) wo da ni (我打你)

yo pegar tú

"te pego"

20) ni da wo (你打我)

tú pegar yo

"me pegas"

21) xia yu (下雨)

caer lluvia

"llueve"

En el chino mandarín, una lengua que utiliza al máximolos recursos de la posición respectiva de los elementos lingüísticos, los participantes de la estructura argumental serán marcados correspondientemente por su anteposición y su posposición con respecto al predicado. Por consiguiente, en (19) y (20), donde $d a$ es el verbo transitivo, los participantes wo y ni son, el agente que controla la acción o el paciente que recibe la acción dependiendo de su posición respecto al verbo. Sin embargo, el argumento que se pospone no siempre es el objeto directo del predicado. En (21), xia yu literalmente significa caer-lluvia. El elemento después del verbo caer no es el objeto que recibe la acción del predicadoverbal sinountemaqueexperimentalaacción decaída, una construcción inacusativa, idéntica a la que tenemos en castellano. Además, los doselementos seusanjuntos comoun verbointransitivo para indicar el fenómeno en que cae agua de las nubes, e igual que la palabra llover, xia yu tampoco permite otro argumento.

Volvamos a la estructura argumental para entender las características léxico-sintácticas de estas dos clases de verbos intransitivos. 
Ambos tipos requieren un solo argumento cuya realización sintáctica es la de sujeto, como los siguientes verbos:

22) llorar, reír, saltar, toser (inergativos)

23) existir, aparecer, llegar, florecer, crecer (inacusativos)

En los siguientes ejemplos se observa la realización semántica de estos verbos en la oración:

24) El niño saltó.

25) El rosal floreció.

En 24) la actividad de saltar depende de la voluntad del sujeto el niño, que sirve de agente en la estructura argumental. En cambio, en 25) el rosal no se puede considerar como instigador del eventoflorecer, sino como un argumento que sufre la acción expresada por el verbo. De esta manera se concluye que en 24) el verbo saltar, que es inergativo, lleva un sujeto de la estructura profunda y que en 25) el verbo inacusativo florecer, que denota estado o evento no agentivo lleva un objeto de la estructura profunda. Levin y Rappaport Hovov describen estas configuraciones sintácticas de la E-P con los siguientes esquemas ${ }^{22}$.

26) Verbo inergativo: $N P\left[{ }_{V P} V\right]$

27) Verbo inacusativo: $\left[{ }_{\mathrm{VP}} \mathrm{VNP} / \mathrm{CP}\right]$

En la estructura argumental, el verbo inergativo lleva un argumento externo en vez de un argumento interno directo. Mientras, el verbo inacusativo tiene un argumento interno directo, papel que

22 Levin y Rappaport Hovov. 
puede desempeñar una cláusula mínima o un sintagma nominal. La diferencia entre el argumento externo y el interno es que el interno recibe la acción o en él se produce o manifiesta la eventualidad que denota el verbo. Así, el externo es agentivo, mientras que el interno es un tema o paciente.

Según el filósofo húngaro Vendler, existe una correlación entre las nociones aspectuales como la telicidad y la inacusatividad; en Verbs and Times $^{23}$, demuestra cuatro categorías verbales:

28) A was running at time t. (activity)

29) A was drawing a circle at t. (accomplishment)

30) A won a race between $t_{1}$ and $t_{2}$. (achievement)

31) A loved somebody from $t_{1}$ to $t_{2}$. (state)

Aplicando las plantillas de estructura de eventos(LSR, Lexical and Semantic Representation) citadas en el trabajo de Mendikoetxea ${ }^{24}$, las cuatro categorías de los verbos se pueden expresar de la siguiente manera:

\section{2) Las plantillas}

a. $\left[\mathrm{x}\right.$ ACT $\left.\mathrm{CMANNER}_{<\mathrm{C}}\right]$ actividad

b. $[\mathrm{x}\langle\mathrm{STATE}\rangle]$ estado

c. $[\mathrm{BECOME}[\mathrm{x}<\mathrm{STATE}>]]$ logro

d. [[x ACT $\left.{ }_{<M A N N E R>}\right]$ CAUSE [BECOME $\left.\left.[\mathrm{y}<\mathrm{STATE}>]\right]\right]$ realización

23 Z. Vendler, Linguistics in Philosophy (Londres: Cornell University Press, 1967).

24 Amaya Mendikoetxea, «En busca de los primitivos léxicos y su realización sintáctica: del léxico a la sintaxis y viceversa», 2 Xarxa Temática de gramática teórica (Barcelona: 2004). 
La representación léxico-semántica se compone de dos elementos primitivos: los predicados primitivos, que forman un conjunto fijo, y las constantes, que pertenecen a un conjunto limitado de tipos semánticos. Tres $(\mathrm{a}, \mathrm{b}, \mathrm{c})$ de estos cuatros tipos de verbos, que tienen un solo argumento, exceptuando verbos de realización (d) de dos argumentos, son los verbos intransitivos. Los verbos de actividad normalmente pertenecen a los inergativos mientras que los verbos de estado y de logro son inacusativos. Sin embargo, es necesario conocer y entender la inacusatividad dentro del contexto concreto sintáctico y semántico. Tomemos el ejemplo del verbo de movimiento correr y su comportamiento variable.

\section{3) Juan corrió. (atélico)}

34) Juan corrió a casa. (télico)

En 33) se puede decir que es un verbo agentivo, atélico, y por lo tanto, de actividad, es decir, un verbo inergativo típico. Sin embargo, en 34) se añade a casa, un adjunto preposicional, con lo que el verbo adquiere un punto final de movimiento. En otras palabras, el acto ya se logró, convirtiendo así la construcción correr a casa en inacusativa. Me asiste la opinión de Noriko, para quien una gradualidad y continuidad desde el extremo de un verbo típicamente inergativo al otro, inacusativo, después de estudiar una serie de verbos en inglés ${ }^{25}$.

35) Unergative

Activities

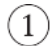

(2)

work cough run
Achievements

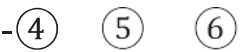

roll break arrive
Unaccusative

States

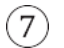

(8) (9) adj. appear stand exist

25 UE Noriko, «On Unergative and Unaccusative Verbs in English» (龙谷大学论集, 2009) 128. 
Hay una clase de verbos en inglés como arrive, ascend, come, exit, etc., que se consideran verbos de dirección inherente. El significado de estos verbos incluye una especificación de la dirección del movimiento. A diferencia de ellos, los verbos run y roll no conllevan la dirección inherente semánticamente. Según Noriko, los verbos de movimiento en general tienen dos componentes semánticos, el propio movimiento y la dirección. El verbo run da más peso al movimiento mientras que el verbo roll da más peso a la dirección, hecho que los coloca en diferentes tipos de verbos intransitivos.

\section{Aspectos sintácticos y semánticos de la inacusatividad}

Según el marco de las teorías generativistas, se distinguen dos tipos de verbos intransitivos. Para muchos lingüistas y sociolingüistas, la adquisición de la inacusatividad en el aprendizaje de una segunda lengua supone un problema de pobreza del estímulo, ya que se cree que cuando los niños aprenden un idioma, a diferencia de los alumnos de L2, tienen acceso a una relativamente gran cantidad de datos lingüísticos. Se considera que el conocimiento de la gramática es suplementado con alguna forma de capacidad lingüística innata. Para un hispanohablante nativo los diferentes usos morfosintácticos que se manifiestan en los ejemplos 36) a 47) son evidentes y naturales. Sin embargo, no todos los nativos son capaces de justificar la gramaticalidad o agramaticalidad sin un buen entendimiento de aspectos sintácticos y semánticos de la inacusatividad; de ahí que convenga la introducción del concepto de inacusatividad en el aula de E/LE. En un trabajo sobre la capacidad de aprendizaje y la inacusatividad, Montrul $^{26}$ señala que con su experimento se descubre que los estudiantes de E/LE parecen tener la capacidad de distinguir sintácticamente los verbos inacusativos e inergativos, por lo menos en algunas construcciones estudiadas, así como que el conocimiento de la inacusatividad

26 S. Monstrul, «On Knowledge and Development of Unaccusativity in Spanish L2 Acquisition», Linguistics 43, 6 (2005): 1153-1190. 
emerge en la etapa intermedia y se adquiere de manera fiable en el nivel avanzado. Compartimos esta opinión y estimamos más adecuado introducir la inacusatividad en el nivel de $\mathrm{C} 1$ o $\mathrm{C} 2$ del marco común europeo de referencia para las lenguas.

36) a. Una vez salido el sol, nos entraremos a la mar.

b. *Trabajado Juan, se dirigió a su casa.

37) a. Un tesoro recientemente aparecido.

b. *Un perro muy ladrado.

38) a. Siempre vienen mujeres.

b. Todos los años llegan cigüeñas.

c. Existen problemas.

39) a. ?Duermen mujeres.

b. ?Juegan niños.

c. ?Trabajan jóvenes.

40) a. Aquí falta (el) café.

b. Aquí sobran (los) fantasmas.

41) a. En el bosque existen hadas y enanitos.

b. En esta casa falta alegría.

c. En este país sobran fantasmas.

d. En la universidad perduran los problemas.

42) a. *En el bosque existen las hadas y enanitos.

b. *En la ciudad hay la corrupción. 
43) a. Escribir-escritor

b. Trabajar-trabajador; correr-corredor; gesticular-gesticulador

c. ${ }^{*}$ venir $\rightarrow$ venidor; ${ }^{*}$ morir $\rightarrow$ moridor; ${ }^{*}$ partir $\rightarrow$ partidor; $*$ salir $\rightarrow$ salidor

44) a. el fabricante de muñecas

b. el cantante de óperas

c. *el muriente

45) a. Los niños jugaron a un juego muy divertido.

b. Juan ríe la risa de un niño.

c. Anoche soñé un sueño aterrador.

46) a. *Esta tribu existe una existencia pacífica.

b. *Pedro apareció una aparición súbita.

47) $\quad$ estar $/ *$ ser crecido, florecido, envejecido

Para explicar las nociones gramaticales de las manifestaciones morfosintácticas, es imprescindible estudiar con más profundidad los aspectos tanto sintácticos como semánticos de la inacusatividad. Para Perlmutter, la inacusatividad viene determinada por la semántica del verbo y aparece codificada en su sintaxis. Aunque la formulación de la hipótesis de la inacusatividad se basa en un estudio de los verbos en inglés, para dar cuenta de la variación interlingüística, muchos lingüistas toman como ejemplo las lenguas romances al estudiar el comportamiento verbal de la inacusatividad. Los trabajos de Burzio ofrecen marcas morfosintácticas claras que sirven de diagnósticos de la inacusatividad. Pero como podemos observar en (36-47), hay pruebas de carácter general que sirven para distinguir los verbos inacusativos de los inergativos en español. Analizando estos ejemplos, podemos resumir algunas observaciones útiles para la distinción de los verbos inergativos: 
(a) Los participios de verbos inacusativos pueden aparecer en cláusulas de participio absoluto, mientras que los verbos inergativos no permiten esta construcción. Los participios adjetivales pueden actuar como modificadores de un SN con función de sujeto sintáctico de un verbo inacusativo, pero no como modificadores del sujeto sintáctico de un verbo inergativo (36-37).

(b) El sujeto sintáctico de un verbo inacusativo puede ser un SN sin determinante posverbal. Esta posibilidad no es aceptada para los verbos inergativos. Las construcciones de inversión locativa sirven a menudo de prueba de ciertos verbos inacusativos (38-42).

(c) El sufijo -dor o -tor o la forma del participio presente -ante que llevan implícita la idea de un agente es compatible con verbos transitivos e verbos inergativos, pero no con los verbos inacusativos (43-44).

(d) Algunos verbos inergativos permiten un complemento tautológico, formando una transitividad cognada, mientras que los verbos inacusativos no admiten objetos cognados (45-46).

(e) Algunos verbos inacusativos permiten la construcción estar + participio para expresar el estado resultante (47).

La observación (a) prueba la distinción de la telicidad entre el verbo inergativo e inacusativo. La Gramática descriptiva, citando el comentario de De Miguel (1992), dice que «la formación de cláusulas de participio sólo es posible con verbos inacusativos que expresan estado final y resultado ${ }^{27}$. Es fácil entender que el participio en (36) expresa una acción télica. Como el inergativo trabajar es un verbo de actividad atélica, no es aceptable el uso de su participio trabajado con el significado de realizar una actividad física o intelectual en general de forma continuada y recibir un salario por ello. Asíque para un hispanohablante nativo suena perfectamente el enunciado como "Llegados los invitados, ..." mientras que no se acepta la estructura * Ladrado el perro. Aquí cabe mencionar otra estructura como Comida la manzana. Sintácticamente se nota la semejanza entre estructuras compuestas por el verbo inacusativo 
llegar y el verbo transitivo comer, e incluso existe concordancia entre el verbo y su argumento correspondiente (llegar-los invitados; comer-la manzana). En su E-P el argumento del verbo inacusativo es el agente, mientras que el argumento del verbo transitivo en esta construcción es el paciente, de ahí que exprese unapasividad.

La observación (b) sirve con frecuencia de prueba de la inacusatividad de los verbos de existencia y aparición. En este tipo de construcción los verbos son «de apoyo» desde el punto de vista de la información, y el orden de palabras es obligatorio en las oraciones de (41). En la construcción de inversión locativa, el argumento locativo se interpreta como el sujeto lógico y se predica la existencia de algo en este lugar. Así, es aceptable un SN sin determinante con una lectura existencial. Este principio es muy importante para aclarar algunas confusiones, ya que en el aula de E/LE se suele destacar que i) el orden del S-V-O en el español no es tan estricto y fijo como en el inglés y el chino; y que ii) en el español, cuando un SN desempeña el papel sintáctico del sujeto en un enunciado, es necesaria la presencia del determinante, contrario a lo que sucede en el inglés y español: life is short y la vida es un sueño.

La observación (c) refuerza el hecho de que los verbos inacusativos tienen sujetos no-agentivos, es decir, en vez de agente, son temas en la posición de argumento interno en la E-P. Así que en 43) y 44), los verbos inergativos y verbos transitivos que son agentivos admiten los mismos cambios morfosintácticos: Juan escribe y puede ser escritor; Juan corre y puede ser corredor; Juan sale pero no será *salidor o *salitor.

La observación (d) se cumple también en inglés: los verbos inacusativos no pueden participar en las construcciones de objeto cognado. En las oraciones de (45), los verbos inergativos demuestran el carácter de verbos transitivos con un objeto sintáctico. Sin embargo, eso no cambia la intransitividad de estos verbos. Por ejemplo, en construcciones como morir una muerte piadosa y vivir una vida feliz, morir y vivir siguen siendo verbos intransitivos. 
La observación (e) lleva a reflexionar sobre el hecho de que los verbos inacusativos no forman una clase semántica homogénea. Los verbos que aparecen en 47) crecer, florecer, envejecer expresan semánticamente un cambio de estado. En la Gramática descriptiva (RAE, 1999: 1583) se mencionan dos tipos de verbos inacusativos: los denominados de cambio de estado o ubicación 48) y los llamados de existencia y aparición 49). Estos dos tipos de verbos pertenecen a la clase de los verbos inacusativos debido a que su sujeto sintáctico es su objeto nocional.

48) romper(se), abrir(se), hundir(se), secar(se), crecer, hervir, palidecer, florecer, caer, levantar(se)

49) aparecer, llegar, existir, ocurrir, venir, emerger, suceder

Evidentemente, la construcción estar + p.p. tiene más compatibilidad con los verbos de 48) que los de 49). Las diferencias semánticas de estos verbos dentro del marco de verbos inacusativos se pueden notar a través de la manifestación en su sintaxis. Por ejemplo, el efecto de inversión locativa sucede si se trata de los verbos de existencia y aparición. Con mucha frecuencia se usan como ejemplos los verbos de cambio de estado como romper(se) y abrir(se) para explicar la alternancia causativa de estos verbos inacusativos. En el trabajo de Levin y Rappaport $\operatorname{Hovov}^{28}$, la alternancia causativa es una de las evidencias más claras de la inacusatividad en inglés. Como hemos notado al principio del presente trabajo, respecto a los enunciados (1) y (2), el verbo open en inglés, “开”en chino y abrir(se) en español, en 1) es intransitivo, mientras que en 2) es transitivo. Su representación léxico-semántica se observa en la siguiente estructura:

50) abrir [[x ACTÚA] CAUSAR [DEVENIR [ $y<$ ABIERTO>]]51)

28 Levin y Rappaport Hovov. 
La ausencia de la variable $x$ muestra sintácticamente el uso intransitivo del verbo, tal como aparece en 1); la coexistencia de las variables tanto de $x$ como de $y$ convierte el verbo intransitivo en un verbo transitivo. Es interesante observar el fenómeno translingüístico de que existe cambio cero durante la alternancia causativa en inglés y en chino, mientras que en español es imprescindible la pronominalización del verbo añadiendo el pronombre se. Ahí se nota una distancia lingüística entre el chino o el inglés, por un lado, y español, por el otro, que servirá para facilitar la adquisición del español como lengua extranjera para los estudiantes chinos con conocimientos previos de la lenguainglesa.

De esta manera, sensibilizar a los estudiantes sobre el fenómeno de la inacusatividad y sus diferencias semánticas y sintácticas en las lenguas facilita un mejor entendimiento de algunos fenómenos gramaticales que se encuentran durante el proceso del aprendizaje de E/LE.

\section{Conclusión}

Hasta ahora no existe en español un estudio detallado sobre la inacusatividad de los verbos como el que proporcionan muchos lingüistas estadounidenses y europeos para el inglés. La Gramática descriptiva de la Real Academia Española ofrece un análisis autoritario sin ser exhaustivo en esta área de la gramática. En el aula de E/LE la inacusatividad es un fenómeno lingüístico poco conocido, a pesar de que la hipótesis de la inacusatividad data de 1978.

Sostenemos la idea de que, con las teorías de la estructura argumental, se puede explicar la agramaticalidad de muchos problemas lingüísticos que surgen durante el proceso del aprendizaje del idioma. La introducción de la dicotomía entre inergativos e inacusativos en la clase de verbos intransitivos, por lo tanto, se vislumbra como una herramienta indispensable para el aprendizaje de español como lengua extranjera, sobre todo, en la etapa avanzada. 